TRANSACTIONS OF THE

AMERICAN MATHEMATICAL SOCIETY

Volume 360, Number 11, November 2008, Pages 5667-5682

S 0002-9947(08)04612-6

Article electronically published on June 19, 2008

\title{
CONTROLLABILITY PROPERTIES OF NONLINEAR BEHAVIORS
}

\author{
FRITZ COLONIUS AND WOLFGANG KLIEMANN
}

\begin{abstract}
This paper proposes a topological framework for the analysis of the time shift on behaviors. It is shown that controllability is not a property of the time shift, while chain controllability is. This also leads to a global decomposition of behaviors.
\end{abstract}

\section{INTRODUCTION}

The analysis of differential equations and, more generally, dynamical systems, via the time shift on a space of trajectories, is a classical approach going back at least to the work of Bebutov 1 in 1940, and it has fostered the development of topological dynamics; compare Sell [15. In control theory, the analysis of input and output functions has a long tradition. A new paradigm, called the behavioral approach to control, has been introduced by Willems [17] and considers systems interacting with the environment without making a difference between inputs and outputs; see also the introductory book [10] by Polderman and Willems. However, so far, this latter theory has essentially been restricted to an algebraic framework. The present paper aims at the analysis of behaviors via topological dynamics of the time shift. It turns out that the basic notion of controllability does not directly lend itself to such an analysis. However, a weakened version, chain controllability, is intimately related to the classical notion of chain transitivity in topological dynamics (see, e.g., Easton [6], Robinson [12]). Additional assumptions allow us to infer controllability from chain controllability.

Our initial goa 1 was to generalize the state space theory of control sets (i.e., of maximal controllable subsets) to input-output systems. This entails that instead of control flows (on the state space of the control system together with the input functions [2] 3]), pairs of input and output functions have to be considered. Now, in this setting, the difference between inputs and outputs turns out to be irrelevant. Thus it appears to us that behaviors provide an appropriate point of view. Clearly, this framework is much more general, and includes, in particular, many implicit systems. However, we will not pursue this direction in the present paper.

The contents are as follows: In Section 2, we consider shift invariant subsets of $L_{\infty}$ endowed with the weak* topology. Restricted to compact subsets, the shift is continuous, and we define and analyze controllable and chain controllable subsets

Received by the editors March 21, 2005.

2000 Mathematics Subject Classification. Primary 37N35, 93B05.

Key words and phrases. Nonlinear control systems, controllability, behaviors.

${ }^{1} \mathrm{~A}$ preliminary version of this paper appeared in [4.

(C)2008 American Mathematical Society Reverts to public domain 28 years from publication 5667 
in this context. In particular, maximal chain controllable sets are characterized as maximal chain transitive sets. In Section 3 topological behaviors are defined via filtrations in $L_{\infty}$. A regular growth condition is used to show that, generically, controllability is equivalent to chain controllability.

\section{Control Sets And Chain COntrol Sets For the time Shift}

In this section, we study controllability properties for the time shift on subsets of $L_{\infty}$-spaces.

Let the time domain $\mathbb{T}$ be equal to $\mathbb{R}$ or $\mathbb{Z}$. Fix a $\sigma$-finite measure $\mu$ on the Lebesgue $\sigma$-algebra in $\mathbb{R}$ and define the time shift $\Theta$ by

$$
\Theta: \mathbb{T} \times L_{\infty}\left(\mathbb{T}, \mathbb{R}^{d}, \mu\right) \rightarrow L_{\infty}\left(\mathbb{T}, \mathbb{R}^{d}, \mu\right),(t, w) \mapsto\left(\Theta_{t} w\right)(s)=w(t+s), s \in \mathbb{T} .
$$

A set $\mathcal{B} \subset L_{\infty}\left(\mathbb{T}, \mathbb{R}^{d}, \mu\right)$ is shift or $\Theta$-invariant if $w \in \mathcal{B}$ implies $\Theta_{t} w \in \mathcal{B}$ for every $t \in \mathbb{T}$. Recall that $L_{\infty}\left(\mathbb{T}, \mathbb{R}^{d}, \mu\right)$ is dual to $L_{1}\left(\mathbb{T}, \mathbb{R}^{d}, \mu\right)$ and that the weak ${ }^{*}$ topology on $L_{\infty}\left(\mathbb{T}, \mathbb{R}^{d}, \mu\right)$ is the weakest topology such that for all $\alpha \in L_{1}\left(\mathbb{T}, \mathbb{R}^{d}, \mu\right)$ the maps

$$
L_{\infty}\left(\mathbb{T}, \mathbb{R}^{d}, \mu\right) \rightarrow \mathbb{R}, w \mapsto \int_{\mathbb{T}} w(t)^{T} \alpha(t) \mu(d t)
$$

are continuous. Fixing a countable dense subset $\left(\alpha_{i}\right) \subset L_{1}\left(\mathbb{T}, \mathbb{R}^{d}, \mu\right)$, the restriction of the metric

$$
\mathrm{d}(v, w)=\sum_{i=1}^{\infty} 2^{-i} \frac{\left|\int_{\mathbb{T}}[v(t)-w(t)]^{T} \alpha_{i}(t) \mu(d t)\right|}{1+\left|\int_{\mathbb{T}}[v(t)-w(t)]^{T} \alpha_{i}(t) \mu(d t)\right|}
$$

to a norm-bounded subset of $L_{\infty}\left(\mathbb{T}, \mathbb{R}^{d}, \mu\right)$ induces the weak* topology. Note also that every weak* compact subset of $L_{\infty}\left(\mathbb{T}, \mathbb{R}^{d}, \mu\right)$ is bounded.

Obviously, the time shift $\Theta$ on $L_{\infty}\left(\mathbb{T}, \mathbb{R}^{d}, \mu\right)$ defines a dynamical system, since $\Theta_{t+s}=\Theta_{t} \circ \Theta_{s}$ for all $s, t \in \mathbb{T}$ and $\Theta_{0}=$ id. The following proposition shows that the restriction to compact subsets is continuous. It follows from continuity of the shift in $L_{1}$.

Proposition 2.1. Let $\mathcal{K}$ be a weak* compact subset of $L_{\infty}\left(\mathbb{T}, \mathbb{R}^{d}, \mu\right)$. Then the restriction of the time shift $\Theta: \mathbb{T} \times \mathcal{K} \rightarrow L_{\infty}\left(\mathbb{T}, \mathbb{R}^{d}, \mu\right)$ is weak $k^{*}$ continuous.

In the following we suppose that a shift invariant subset $\mathcal{B} \subset L_{\infty}\left(\mathbb{T}, \mathbb{R}^{d}, \mu\right)$ is given. We define controllability for the time shift by adapting a proposal by Jan Willems to our situation.

Definition 2.2. For $v \in \mathcal{B}$ the positive orbit at time $T>0$ is defined as

$$
\mathcal{O}_{T}^{+}(v)=\left\{w \in \mathcal{B}, \text { there is } w_{1} \in \mathcal{B} \text { with } w_{1}(t)=\left\{\begin{array}{cl}
v(t) & \text { for } \quad t \leq 0 \\
w(t-T) & \text { for } \quad t \geq T
\end{array}\right\},\right.
$$

and the positive orbit is defined by

$$
\mathcal{O}^{+}(v)=\bigcup_{T>0} \mathcal{O}_{T}^{+}(v)
$$

Of particular interest are subsets of complete controllability defined as follows.

Definition 2.3. A (behavioral) control set is a nonvoid maximal shift invariant subset $\mathcal{D} \subset \mathcal{B}$ with $\mathcal{D} \subset \mathcal{O}^{+}(v)$ for all $v \in \mathcal{D}$. 
More explicitly, a nonvoid shift invariant subset $\mathcal{D}$ is a control set if for all $v, w \in \mathcal{D}$ there are $w_{1} \in \mathcal{B}$ and a time $T>0$ satisfying

$$
w_{1}(t)=\left\{\begin{array}{cc}
v(t) & \text { for } \quad t \leq 0 \\
w(t-T) & \text { for } \quad t \geq T
\end{array}\right.
$$

and every set $\mathcal{D}^{\prime}$ with $\mathcal{D} \subset \mathcal{D}^{\prime} \subset \mathcal{B}$ with this property satisfies $\mathcal{D}^{\prime}=\mathcal{D}$. Using maximality, it is easy to show that $w_{1}$ with the property above is also in $\mathcal{D}$. Note also that the controllability property is required within the set $\mathcal{B} \subset L_{\infty}\left(\mathbb{T}, \mathbb{R}^{d}, \mu\right)$.

As an example we consider input-output pairs of continuous-time, control-affine systems in $\mathbb{R}^{n}$ described by

$$
\begin{aligned}
& \dot{x}(t)=f_{0}(x(t))+\sum_{i=1}^{m} u_{i}(t) f_{i}(x(t)), \\
& y(t)=h(x(t), u(t))=h_{0}(x(t))+\sum_{i=1}^{m} u_{i}(t) h_{i}(x(t)),
\end{aligned}
$$

with inputs $\left(u_{i}\right)$ taking values in $\mathbb{R}^{m}$; furthermore, the $f_{i}$ are smooth $\left(C^{\infty}-\right)$ vector fields and the output functions $h_{i}: \mathbb{R}^{n} \rightarrow \mathbb{R}^{k}$ are also smooth. Assume that for every $x \in \mathbb{R}^{n}$ and every input $u \in L_{\infty}\left(\mathbb{R}, \mathbb{R}^{m}\right)$ (here we take the Lebesgue measure $\lambda$ on $\mathbb{R}$ ) there exists a unique absolutely continuous global solution $\varphi(t, x, u), t \in \mathbb{R}$. Also denote

$$
\mathcal{U}=\left\{u \in L_{\infty}\left(\mathbb{R}, \mathbb{R}^{m}\right), u(t) \in U \text { for almost all } t \in \mathbb{R}\right\},
$$

where $U$ is a subset of $\mathbb{R}^{m}$.

Proposition 2.4. Consider the input-output system (2). For every compact convex set $U \subset \mathbb{R}^{m}$ and every compact set $K \subset \mathbb{R}^{n}$ the set

$$
\mathcal{B}_{U \times K}=\left\{(u, y) \in L_{\infty}\left(\mathbb{R}, \mathbb{R}^{m+k}\right), \begin{array}{c}
u \in \mathcal{U} \text { and there is } x \in \mathbb{R}^{n} \text { s.t. for } t \in \mathbb{R} \\
\varphi(t, x, u) \in K \text { and } y(t)=h(\varphi(t, x, u), u(t))
\end{array}\right\}
$$

is weak* compact and shift invariant.

Proof. Shift invariance is clear by definition. The set $\mathcal{U} \subset L_{\infty}\left(\mathbb{R}, \mathbb{R}^{m}\right)$ is weak* compact and the map

$$
(t, x, u) \mapsto(\varphi(t, x, u), u(t+\cdot)): \mathbb{R} \times K \times \mathcal{U} \rightarrow K \times \mathcal{U}
$$

is continuous, uniformly on bounded intervals in $\mathbb{R}$. Then the desired compactness follows, since $K$ is compact and $h$ is control affine.

Remark 2.5. For control-affine systems (2), a control set $D$ with nonvoid interior in the state space $\mathbb{R}^{n}$ is defined as a maximal set of approximate controllability. If the system is locally accessible (i.e., the sets of points reachable up to time $\pm T$ from points $x$ have nonvoid interiors for every $T>0$ ), exact controllability in the interior follows, and one easily sees that the following set is contained in a control set $\mathcal{D}$ in the sense of Definition 2.3:

$$
\left\{(u, y) \in \mathcal{B}, \begin{array}{l}
\text { there is } x_{0} \in \operatorname{int} D \text { with } \varphi\left(t, x_{0}, u\right) \in \operatorname{int} D \\
\text { and } y(t)=h\left(\varphi\left(t, x_{0}, u\right), u(t)\right) \text { for all } t \in \mathbb{R}
\end{array}\right\} .
$$

In fact, the controllability property is immediate. For input-state systems (i.e., $y=x)$ note the difference to the so-called lift of a control set where, instead of input-trajectory pairs, the closure of the set of pairs $\left(u, x_{0}\right) \in \mathcal{U} \times \operatorname{int} D$ with $\varphi\left(t, x_{0}, u\right) \in \operatorname{int} D$ for all $t$ is considered. 
Example 2.6. Consider the linear control system

$$
\dot{x}(t)=A x(t)+B u(t), y(t)=C x(t)+D u(t)
$$

with matrices $A, B, C, D$ of appropriate dimension and with $u(t) \in U$, for a compact and convex subset $U \subset \mathbb{R}^{m}$ containing the origin in its interior. Here it is known (see Colonius and Spadini [5]) that there exists a unique bounded control set in $\mathbb{R}^{n}$ if the pair $(A, B)$ is controllable and $A$ is hyperbolic. This implies that for $K \subset \mathbb{R}^{n}$ large enough, the system (3) has a behavioral control set obtained as in Remark 2.5.

For control-affine state space systems, a control set $D$ with nonvoid interior in $\mathbb{R}^{n}$ can be characterized as the projection to $\mathbb{R}^{n}$ of a topologically mixing set of the associated dynamical system on $\mathcal{U} \times \mathbb{R}^{n}$ provided that local accessibility holds. Certainly, not all topologically mixing sets in $L_{\infty}\left(\mathbb{R}, \mathbb{R}^{d}\right)$ are control sets (consider, e.g., a quasi-periodic motion on the 2 -torus). Nevertheless, behavioral control sets turn out to be topologically mixing.

Proposition 2.7. Let $\mathcal{D} \subset L_{\infty}\left(\mathbb{T}, \mathbb{R}^{d}, \mu\right)$ be a control set with diamD $<\infty$. Then $\mathcal{D}$ is topologically mixing.

Proof. We have to show that for every pair $W_{1}, W_{2}$ of open sets in $\mathcal{D}$ there is $T_{0}>0$ such that $\Theta_{-T_{0}}\left(W_{1}\right) \cap W_{2} \neq \emptyset$. It suffices to consider sets in a subbasis of the weak* topology. Hence we may assume that for $j=1,2$ the set $W_{j}$ has the form $W_{j}=V_{j} \cap \mathcal{D}$ with

$$
V_{j}=\left\{v \in L_{\infty}\left(\mathbb{T}, \mathbb{R}^{d}, \mu\right),\left|\int_{\mathbb{R}}\left\langle v_{j}(t)-v(t), y_{i j}(t)\right\rangle \mu(d t)\right|<\varepsilon \text { for } i=1, \ldots, k_{j}\right\}
$$

for $v_{j} \in \mathcal{D}$ and functions $y_{i j} \in L_{1}\left(\mathbb{T}, \mathbb{R}^{d}, \mu\right)$. There is $T>0$, such that for $j=1,2$ and $i=1, \ldots, k_{j}$

$$
\int_{\mathbb{R} \backslash[-T, T]}\left|y_{i j}(t)\right| \mu(d t)<\frac{\varepsilon}{\operatorname{diam\mathcal {D}}} .
$$

Since $\Theta_{-T}\left(v_{1}\right), \Theta_{T}\left(v_{2}\right) \in \mathcal{D}$, there are $t_{0}>0$ and $v_{0} \in \mathcal{D}$ with

$$
v_{0}(t)= \begin{cases}v_{2}(T+t) & \text { for } t \leq 0 \\ v_{1}(t-T) & \text { for } t \geq t_{0}\end{cases}
$$

Then $v:=\Theta_{-T} v_{0} \in \mathcal{D}$, and with (4) one sees that $v \in V_{2}$ and $v\left(t_{0}+2 T+\cdot\right) \in V_{1}$. Thus $v \in W_{2}$ and $v\left(t_{0}+2 T+\cdot\right) \in W_{1}$, and the assertion follows with $T_{0}:=$ $t_{0}+2 T$.

The definition of a behavioral control set requires that one can precisely 'hit' the function $w$ after some time. It may appear natural to introduce a weaker concept, in analogy to chain controllability in the state space. As it turns out, this concept leads to sets that are 'better behaved' from a topological point of view, just as chain control sets are in the state space setup. Observe again that the definition below is not given in the flow context; it is strictly analogous to the definition of (behavioral) control sets. First, a notion of approximate controllability is obtained by requiring that the following semi-distance on $L_{\infty}\left(\mathbb{T}, \mathbb{R}^{d}, \mu\right)$,

$$
\mathrm{d}^{+}(v, w)=\mathrm{d}\left(\chi_{[0, \infty)} \cdot v, \chi_{[0, \infty)} \cdot w\right),
$$


is small; here $\chi_{[0, \infty)}$ is the characteristic function of $[0, \infty)$. Thus this semi-distance only takes into account the positive time set. We define sets of chain controllability in the following way.

Definition 2.8. For $\varepsilon, T>0$ an $(\varepsilon, T)^{+}$-chain from $v \in \mathcal{B}$ to $w \in \mathcal{B}$ is given by

$$
n \in \mathbb{N}, w_{0}=v, w_{1}, \ldots, w_{n}=w \in \mathcal{B}, T_{0}, \ldots, T_{n-1} \geq T,
$$

such that

$$
\mathrm{d}^{+}\left(\Theta_{T_{i}}\left(w_{i}\right), w_{i+1}\right)<\varepsilon \text { for all } i .
$$

If for all $\varepsilon, T>0$ there is an $(\varepsilon, T)^{+}$-chain from $v \in \mathcal{B}$ to $w \in \mathcal{B}$, we say that $v$ is chain controllable to $w$. The chain orbit of $v \in \mathcal{B}$ is

$$
\mathcal{O}_{c}^{+}(v):=\left\{w \in \mathcal{B} \text {, for all } \varepsilon>0 \text { there is an }(\varepsilon, T)^{+}-\text {chain from } v \text { to } w\right\} .
$$

Note that in the definition of chain orbits $\mathcal{O}_{c}^{+}$, it is equivalent to consider only $(\varepsilon, 1)^{+}$-chains; see, e.g., Szolnoki [16].

We will consider maximal subsets which are chain controllable.

Definition 2.9. A nonvoid invariant subset $\mathcal{E} \subset \mathcal{B}$ is a (behavioral) chain control set if it is a maximal set such that for all $v, w \in \mathcal{E}$ and all $\varepsilon, T>0$ there is an $(\varepsilon, T)^{+}$-chain in $\mathcal{E}$ from $v$ to $w$.

For these sets, contrary to control sets, we will be able to provide a complete characterization in terms of the system $\Theta$. Recall from the theory of dynamical systems (see [12]) that $(\varepsilon, T)$-chains for a continuous flow are defined as in Definition 2.8 but with the semi-distance $\mathrm{d}^{+}$replaced by a distance $\mathrm{d}$ in the metric space. They give rise to chain transitive sets in analogy to Definition 2.9 .

Theorem 2.10. Let a shift-invariant set $\mathcal{B} \subset L_{\infty}\left(\mathbb{T}, \mathbb{R}^{d}, \mu\right)$ be given. A nonempty weak $^{*}$ compact and shift invariant set $\mathcal{E} \subset \mathcal{B}$ is a chain control set if and only if the restriction of the shift to $\mathcal{E}$ is chain transitive and $\mathcal{E}$ is a maximal set with this property, i.e., if $\mathcal{E} \subset \mathcal{E}^{\prime} \subset \mathcal{B}$ and $\mathcal{E}^{\prime}$ is compact and invariant such that the shift restricted to $\mathcal{E}^{\prime}$ is chain transitive, then $\mathcal{E}=\mathcal{E}^{\prime}$.

Proof. Recall the definition of the metric d in (1) and, for $\varepsilon>0$, choose $k \in \mathbb{N}$ large enough such that

$$
\sum_{i=k+1}^{\infty} 2^{-i}<\varepsilon .
$$

For the finitely many $\alpha_{1}, \ldots, \alpha_{k} \in L_{1}\left(\mathbb{T}, \mathbb{R}^{d}, \mu\right)$ there is $S>0$ such that for all $i$

$$
\int_{\mathbb{T} \backslash[-S, S]}\left|\alpha_{i}(\tau)\right| \mu(d \tau)<\frac{\varepsilon}{\operatorname{diam\mathcal {E}}} .
$$

Suppose that $\mathcal{E}$ is a chain control set. Let $v, w \in \mathcal{E}$ and pick $\varepsilon, T>0$. We may assume that $T>S$. Chain controllability from $v$ to $w(-S+\cdot)$ yields the existence of $n \in \mathbb{N}$ and $v_{0}, \ldots, v_{n} \in \mathcal{E}, T_{0}, \ldots, T_{n-1}>T+S$ with $v_{0}=v, v_{n}=w(-S+\cdot)$ and

$$
\mathrm{d}^{+}\left(\Theta_{T_{i}} v_{i}, v_{i+1}\right)<\varepsilon \text { for } j=0, \ldots, n-1 \text {. }
$$

Now construct an $(\varepsilon, T)$-chain from $v$ to $w$ in the following way ('jumping later'). Define

$$
w_{0}=v, w_{j}=\Theta_{S} v_{j} \text { for } j=1, \ldots, n-1, w_{n}=\Theta_{S} v_{n}=w,
$$


and let the jump times be $t_{j}=T_{j}+S$. Then

$$
\begin{aligned}
& \mathrm{d}\left(\Theta_{t_{0}} w_{0}, w_{1}\right)=\mathrm{d}\left(\Theta_{T_{0}+S} v, \Theta_{S} v_{1}\right) \\
& =\sum_{i=1}^{\infty} 2^{-i} \frac{\left|\int_{\mathbb{T}}\left[v\left(t+T_{0}+S\right)-v_{1}(t+S)\right]^{T} \alpha_{i}(t) \mu(d t)\right|}{1+\left|\int_{\mathbb{T}}\left[v\left(t+T_{0}+S\right)-v_{1}(t+S)\right]^{T} \alpha_{i}(t) \mu(d t)\right|} \\
& \leq \sum_{i=1}^{k} 2^{-i} \frac{\left|\int_{\mathbb{T}}\left[v\left(t+T_{0}+S\right)-v_{1}(t+S)\right]^{T} \alpha_{i}(t) \mu(d t)\right|}{1+\left|\int_{\mathbb{T}}\left[v\left(t+T_{0}+S\right)-v_{1}(t+S)\right]^{T} \alpha_{i}(t) \mu(d t)\right|}+\varepsilon .
\end{aligned}
$$

Now for $i=1, \ldots, k$

$$
\begin{aligned}
& \left|\int_{\mathbb{T}}\left[v\left(t+T_{0}+S\right)-v_{1}(t+S)\right]^{T} \alpha_{i}(t) \mu(d t)\right| \\
& \leq \int_{\mathbb{T} \backslash[-S, S]}\left|\alpha_{i}(t)\right| \mu(d t) 2 \operatorname{diam} \mathcal{E}+\left|\int_{-S}^{S}\left[v\left(t+T_{0}+S\right)-v_{1}(t+S)\right]^{T} \alpha_{i}(t) \mu(d t)\right| \\
& <2 \varepsilon+\left|\int_{0}^{2 S}\left[v\left(t+T_{0}\right)-v_{1}(t)\right]^{T} \alpha_{i}(t) \mu(d t)\right|<5 \varepsilon,
\end{aligned}
$$

since by (7) and (6)

$$
\begin{aligned}
& \left|\int_{0}^{2 S}\left[v\left(t+T_{0}\right)-v_{1}(t)\right]^{T} \alpha_{i}(t) \mu(d t)\right| \\
& =\left|\int_{0}^{\infty}\left[v\left(t+T_{0}\right)-v_{1}(t)\right]^{T} \alpha_{i}(t) \mu(d t)-\int_{2 S}^{\infty}\left[v\left(t+T_{0}\right)-v_{1}(t)\right]^{T} \alpha_{i}(t) \mu(d t)\right| \\
& \leq\left|\int_{0}^{\infty}\left[v\left(t+T_{0}\right)-v_{1}(t)\right]^{T} \alpha_{i}(t) \mu(d t)\right|+2 \varepsilon<3 \varepsilon .
\end{aligned}
$$

Thus

$$
\mathrm{d}\left(\Theta_{t_{0}} w_{0}, w_{1}\right)<6 \varepsilon .
$$

Analogously, one shows that $\mathrm{d}\left(\Theta_{t_{j}} w_{j}, w_{j+1}\right)<6 \varepsilon$ for all $j=1, \ldots, n-1$. This proves that the restriction of $\Theta$ to the chain control set $\mathcal{E}$ is chain transitive.

Conversely, suppose that $\mathcal{E}$ is a chain transitive set, and let $v, w \in \mathcal{E}$. By assumption one finds for all $\varepsilon, T>0$ an $(\varepsilon, T)$-chain given by $v_{0}=v, v_{1}, \ldots, v_{n}=\Theta_{S} w$ in $\mathcal{E}$ and $T_{0}, \ldots, T_{n-1}>T$ from $v$ to $w$ with

$$
\mathrm{d}\left(\Theta_{T_{i}} v_{i}, v_{i+1}\right)<\varepsilon
$$

We may assume that conditions (5) and (6) are satisfied and that $T_{j}-S>T$. This gives rise to an $(\varepsilon, T)^{+}$-chain in the following way ('jumping earlier').

Define

$$
w_{0}=v, w_{j}=\Theta_{-S} v_{j} \text { for } j=1, \ldots, n-1, w_{n}=\Theta_{-S} v_{n}=w,
$$


and let the jump times be $t_{j}=T_{j}-S$. Then

$$
\begin{aligned}
& \mathrm{d}^{+}\left(\Theta_{t_{0}} w_{0}, w_{1}\right)=\mathrm{d}^{+}\left(\Theta_{T_{0}-S} v, \Theta_{-S} v_{1}\right) \\
& =\sum_{i=1}^{\infty} 2^{-i} \frac{\left|\int_{0}^{\infty}\left[v\left(t+T_{0}-S\right)-v_{1}(t-S)\right]^{T} \alpha_{i}(t) \mu(d t)\right|}{1+\left|\int_{0}^{\infty}\left[v\left(t+T_{0}-S\right)-v_{1}(t-S)\right]^{T} \alpha_{i}(t) \mu(d t)\right|} \\
& \leq \sum_{i=1}^{k} 2^{-i} \frac{\left|\int_{0}^{\infty}\left[v\left(t+T_{0}-S\right)-v_{1}(t-S)\right]^{T} \alpha_{i}(t) \mu(d t)\right|}{1+\left|\int_{0}^{\infty}\left[v\left(t+T_{0}-S\right)-v_{1}(t-S)\right]^{T} \alpha_{i}(t) \mu(d t)\right|}+\varepsilon .
\end{aligned}
$$

Now for $i=1, \ldots, k$

$$
\begin{aligned}
& \left|\int_{0}^{\infty}\left[v\left(t+T_{0}-S\right)-v_{1}(t-S)\right]^{T} \alpha_{i}(t) \mu(d t)\right| \\
& \leq \int_{2 S}^{\infty}\left|\alpha_{i}(t)\right| \mu(d t) 2 \operatorname{diam} \mathcal{E}+\left|\int_{0}^{2 S}\left[v\left(t+T_{0}-S\right)-v_{1}(t-S)\right]^{T} \alpha_{i}(t) \mu(d t)\right| \\
& <2 \varepsilon+\left|\int_{-S}^{S}\left[v\left(t+T_{0}\right)-v_{1}(t)\right]^{T} \alpha_{i}(t) \mu(d t)\right|<5 \varepsilon,
\end{aligned}
$$

since by (8) and (6)

$$
\begin{aligned}
& \left|\int_{-S}^{S}\left[v\left(t+T_{0}\right)-v_{1}(t)\right]^{T} \alpha_{i}(t) \mu(d t)\right| \\
& =\left|\int_{\mathbb{T}}\left[v\left(t+T_{0}\right)-v_{1}(t)\right]^{T} \alpha_{i}(t) \mu(d t)-\int_{\mathbb{T} \backslash[-S, S]}\left[v\left(t+T_{0}\right)-v_{1}(t)\right]^{T} \alpha_{i}(t) \mu(d t)\right| \\
& \leq\left|\int_{\mathbb{T}}\left[v\left(t+T_{0}\right)-v_{1}(t)\right]^{T} \alpha_{i}(t) \mu(d t)\right|+2 \varepsilon<3 \varepsilon .
\end{aligned}
$$

Thus

$$
\mathrm{d}^{+}\left(\Theta_{t_{0}} w_{0}, w_{1}\right)<6 \varepsilon .
$$

Analogously, one shows that $\mathrm{d}^{+}\left(\Theta_{t_{j}} w_{j}, w_{j+1}\right)<6 \varepsilon$ for all $j=1, \ldots, n-1$.

It only remains to show the maximality properties. A chain control set $\mathcal{E}$ is a maximal chain transitive set: In fact, suppose that the restriction of $\Theta$ to $\mathcal{E}^{\prime} \supset \mathcal{E}$ is chain transitive. Then it follows that $\mathcal{E}^{\prime}=\mathcal{E}$, since chain transitivity of $\mathcal{E}^{\prime}$ implies, as just proven, that $\mathcal{E}^{\prime}$ is chain controllable and $\mathcal{E}$ is a maximal chain controllable set. In the same way, one sees that a chain control set $\mathcal{E}$ is a maximal set with the property that the restriction of $\Theta$ to $\mathcal{E}$ is chain transitive.

Remark 2.11 (On connections). Let $\mathcal{B}^{1}$ and $\mathcal{B}^{2}$ be shift invariant subsets of $L_{\infty}\left(\mathbb{T}, \mathbb{R}^{d}, \mu\right)$ given by input-output pairs $\left(u^{1}, y^{1}\right)$ and $\left(u^{2}, y^{2}\right)$ as in (2). Suppose that $\mathcal{E}^{1}$ and $\mathcal{E}^{2}$ are chain control sets of $\mathcal{B}^{1}$ and $\mathcal{B}^{2}$, respectively, and the intersection (9) $\left\{y^{1}\right.$, there is $u^{1}$ with $\left.\left(u^{1}, y^{1}\right) \in \mathcal{E}^{1}\right\} \cap\left\{u^{2}\right.$, there is $y^{2}$ with $\left.\left(u^{2}, y^{2}\right) \in \mathcal{E}^{2}\right\}$

is nonvoid and compact. Then this set has nonvoid intersection with a chain control set of the connection

$$
\mathcal{B}^{1} * \mathcal{B}^{2}:=\left\{\left(u^{1}, y^{2}\right) \text {, there is } y^{1}=u^{2} \text { with }\left(u^{1}, y^{1}\right) \in \mathcal{B}^{1} \text { and }\left(u^{2}, y^{2}\right) \in \mathcal{B}^{2}\right\} .
$$

This follows since the set in (9) is compact and shift invariant (but not necessarily chain transitive). Hence it contains a chain transitive subset. 
Remark 2.12. For control-affine systems (2) with $y=x$, a chain control set $E$ in the state space $\mathbb{R}^{n}$ is defined as a maximal set such that for all $x \in E$ there is $u \in \mathcal{U}$ with $\varphi(t, x, u) \in E$ for all $t \in \mathbb{R}$, and for all $x, y \in E$ and $\varepsilon, T>0$ there are $T_{0}, \ldots, T_{n-1}>T$, points $x_{0}=x, x_{1}, \ldots, x_{n}=y$, and controls $u_{0}, \ldots, u_{n-1} \in \mathcal{U}$ with $\left|\varphi\left(T_{i}, x_{i}, u_{i}\right)-x_{i+1}\right|<\varepsilon$ for all $i$. These sets correspond to the maximal chain transitive sets of the control flow $(u, x) \mapsto(u(t+\cdot), \varphi(t, x, u))$ on $\mathcal{U} \times \mathbb{R}^{n}$. Using continuity of this flow one shows that these chain control sets $E$ are in $1-1$-correspondence with the behavioral chain control sets $\mathcal{E}$ via

$$
\mathcal{E}=\left\{\left(u, \varphi(\cdot, x, u) \in L_{\infty}\left(\mathbb{R}, \mathbb{R}^{m} \times \mathbb{R}^{n}\right), \varphi(t, x, u) \in E \text { for all } t \in \mathbb{R}\right\} .\right.
$$

Theorem 2.10 identifies the chain control sets as the maximal chain transitive sets. Hence one obtains the following result on global decompositions from Conley's Fundamental Theorem. Here the stable set of a closed shift invariant set $Y$ is defined as

$$
W^{s}(Y)=\left\{w \in \mathcal{B}, \mathrm{d}\left(\Theta_{t} w, Y\right) \rightarrow 0 \text { for } t \rightarrow \infty\right\} .
$$

Corollary 2.13. Let $\mathcal{K}$ be a weak* compact subset of a shift invariant set $\mathcal{B} \subset$ $L_{\infty}\left(\mathbb{T}, \mathbb{R}^{d}, \mu\right)$. Then $\mathcal{K}$ is the disjoint union of the stable sets of the chain control sets with respect to $\mathcal{K}$ together with the set $\mathcal{L}$ of those points in $\mathcal{K}$ leaving $\mathcal{K}$ in positive time.

Proof. For discrete time systems, i.e., $\mathbb{T}=\mathbb{Z}$, this is proved in Easton [6] (with slightly different, but equivalent notions). For $\mathbb{T}=\mathbb{R}$, one has to observe that in a maximal chain transitive set one may take all jump times equal to 1 (the relevant arguments are given, e.g., in Szolnoki [16]). Thus the continuous time case can be reduced to the discrete time case.

We conclude this section with an example illustrating the decomposition property in Corollary 2.13. It is the model of a continuous stirred tank reactor due to Poore [1] (see also Golubitsky and Schaeffer [8] for more information on this system).

Example 2.14. Consider the control-affine system (without output)

$$
\left(\begin{array}{l}
\dot{x}_{1} \\
\dot{x}_{2}
\end{array}\right)=\left(\begin{array}{l}
-x_{1}-a\left(x_{1}-x_{c}\right)+B \alpha\left(1-x_{2}\right) e^{x_{1}} \\
-x_{2}+\alpha\left(1-x_{2}\right) e^{x_{1}}
\end{array}\right)+u(t)\left(\begin{array}{c}
x_{c}-x_{1} \\
0
\end{array}\right) .
$$

Here $x_{1}$ is the (dimensionless) temperature, $x_{2}$ is the product concentration, and $a, \alpha, B$, and $x_{c}$ are positive constants. The parameter $x_{c}$ is the coolant temperature, and hence the control affects the heat transfer coefficient. The parameter values

$$
a=0.15, \alpha=0.05, B=7.0, x_{c}=1.0
$$

guarantee that for constant control $u(t) \equiv 0$ system (10) has exactly three fixed points in the physically relevant region $[0, \infty) \times[0,1] \subset \mathbb{R}^{2}$ as limit sets. They are given by $x^{i}=\left(z^{i}, y^{i}\right)$ where $y^{i}=\alpha e^{z^{i}} /\left(1+\alpha z^{i}\right), i=0,1,2$, and $z^{0}<z^{1}<z^{2}$ are the zeros of the transcendental equation

$$
-z-(a+u)\left(z-x_{c}\right)+B \alpha\left[1-\frac{\alpha e^{z}}{1+\alpha e^{z}}\right] e^{z}=0 .
$$

The fixed points $x^{0}$ and $x^{2}$ are asymptotically stable and $x^{1}=\left(z^{1}, y^{1}\right)$ is hyperbolic. For a compact interval $U \subset \mathbb{R}$ as control range, consider the trajectories that, under all controls $u \in \mathcal{U}$, remain for positive times $t \geq 0$ in the set $K=[0,7] \times[0,1]$ containing the equilibria in its interior. There are chain control sets $E^{i}$ containing 
$x^{i}$. In fact, using Gayer [7, for all but at most countably many $\rho \in[0,0.15]$ the control range $[-\rho, \rho]$ yields that the chain control sets are the closures of control sets containing $x^{i}$ in the interior, and for $\rho \rightarrow 0$ the chain control sets $E^{i}$ converge to $\left\{x^{i}\right\}$. Hence, multiplying the vector fields in (10) by a smooth cut-off function $\chi$ with $\chi\left(x_{1}, x_{2}\right)=0$ iff $\left(x_{1}, x_{2}\right) \notin K$ and $\chi\left(x_{1}, x_{2}\right)=1$ on a closed set containing all chain control sets $E^{i}$ for $\rho \leq 0.15$, we obtain that $K$ is a compact invariant set for the modified system. The corresponding set of input-state pairs

$$
\mathcal{B}_{U \times K}:=\left\{(u, \varphi(\cdot, x, u)) \in L_{\infty}\left(\mathbb{R}, \mathbb{R} \times \mathbb{R}^{2}\right), u \in \mathcal{U} \text { and } x \in K\right\}
$$

is shift invariant and weak ${ }^{*}$ compact. For a chain control set $E^{i}$ contained in the state space $\mathbb{R}^{2}$ (cf. Remark 2.12) denote

$$
\mathcal{E}^{i}=\left\{(u, \varphi(\cdot, x, u)) \in \mathcal{B}_{U \times K}, u \in \mathcal{U} \text { and } x \in E^{i}\right\} .
$$

Then $\mathcal{E}^{i}$ is a chain control set for $\mathcal{B}_{U \times K}$ and the set $\mathcal{B}_{U \times K}$ is the disjoint union of the stable sets of its chain control sets $\mathcal{E}^{i}$. Note that, besides the chain control sets $\mathcal{E}^{i}$ obtained from the chain control sets $E^{i}$ in the state space $K \subset \mathbb{R}^{2}$ there is the artificial chain control set $\partial K$ obtained from the cut-off. One can show that the domain of attraction of the chain control set $E^{1}$ in the state space,

$$
\mathbf{A}^{+}\left(E^{1}\right):=\left\{x \in K, \text { there is } u \in \mathcal{U} \text { with } \varphi(t, x, u) \rightarrow E^{1} \text { for } t \rightarrow \infty\right\},
$$

is given by the union of the stable manifolds $W^{+}(u)$ of the hyperbolic equilibria in $E^{1}$ corresponding to constant controls $u$,

$$
\mathbf{A}^{+}\left(E^{1}\right)=\operatorname{int} K \cap \bigcup_{u \in[-\rho, \rho]} W^{+}(u) .
$$

The left and right boundaries are given by the stable manifolds for $u \equiv-\rho$ and $u \equiv+\rho$, respectively. Then, for the stable set of the chain control set $\mathcal{E}^{1}$ one finds

$$
W^{s}\left(\mathcal{E}^{1}\right)=\left\{(u, \varphi(\cdot, x, u)) \in L_{\infty}\left(\mathbb{R}, \mathbb{R} \times \mathbb{R}^{2}\right), \begin{array}{c}
\varphi(t, x, u)) \in \mathbf{A}^{+}\left(E^{1}\right) \text { for } \\
\text { all } t \geq 0 \text { and } u \in \mathcal{U}
\end{array}\right\}
$$

While, analytically, it is difficult to proceed further, numerical results [3, Section 9.1] indicate the following: No further chain control sets exist and for $t \rightarrow \infty$, points to the left of the right boundary of $\mathbf{A}^{+}\left(E^{1}\right)$ can be steered to $E^{0}$, and points right to the left boundary of $\mathbf{A}^{+}\left(E^{1}\right)$ can be steered to $E^{2}$. This yields the stable sets of $\mathcal{E}^{0}$ and $\mathcal{E}^{2}$, similar to (12).

If we consider a proper subset $K^{\prime}$ of $[0,7] \times[0,1]$, we may obtain a nontrivial set $\mathcal{L}$ of pairs $(u, \varphi(\cdot, x, u))$ leaving $\mathcal{B}_{U \times K^{\prime}}$. For example, we may take $K^{\prime}$ as a compact subset containing $x^{0}$ and $x^{1}$ in its interior, but not containing $x^{2}$. Suppose that $\rho$ is chosen small enough such that the chain control set around $x^{2}$ has void intersection with $K^{\prime}$. Then for every $x \in \mathbf{A}^{+}\left(E^{1}\right)$ there is $u \in \mathcal{U}$ with $\Theta_{t}(u, \varphi(\cdot, x, u)) \notin \mathcal{B}_{U \times K^{\prime}}$ for large $t>0$, and hence $\mathcal{B}_{U \times K^{\prime}}$ is not positively invariant.

\section{TOPOlOGiCAL BeHAVIORS AND REgUlar GROWTH}

In this section, we define topological behaviors and show that under a regular growth condition, chain control sets and control sets generically coincide.

Recall that a quasi-order on a set $A$ is a relation $\leq$ that is reflexive and transitive. As usual, we write $\alpha<\beta$ if $\alpha \leq \beta$ and $\alpha \neq \beta$. The following examples, where the order is given by set inclusion, will be relevant. 
Example 3.1. Let $\operatorname{co}\left(\mathbb{R}^{d}\right)$ denote the family of all compact subsets of $\mathbb{R}^{d}$. For $K, L \in \operatorname{co}\left(\mathbb{R}^{d}\right)$, define a quasi-order by $L \leq K$ if $L \subset K$. Another example is the family $\mathrm{Co}_{0}\left(\mathbb{R}^{d}\right)$ of all compact convex subsets of $\mathbb{R}^{d}$ that contain the origin in their interior. If we fix $K \in \mathrm{Co}_{0}\left(\mathbb{R}^{d}\right)$, the family of sets $K^{\rho}:=\rho \cdot K, \rho>0$, is quasiordered. In product spaces products of these quasi-ordered sets yield quasi-ordered sets.

Now we define the central notion of this paper.

Definition 3.2. A topological behavior $\mathcal{B}$ is a subset of $L_{\infty}\left(\mathbb{T}, \mathbb{R}^{d}, \mu\right)$ together with a filtration $\left\{\mathcal{B}_{\alpha}\right\}_{\alpha \in A}$ in the following sense: The sets $\mathcal{B}_{\alpha}$ are shift invariant and weak* compact in $L_{\infty}\left(\mathbb{T}, \mathbb{R}^{d}, \mu\right)$ and $A$ is quasi-ordered with

$$
\mathcal{B}=\bigcup_{\alpha \in A} \mathcal{B}_{\alpha} \text { and } \alpha \leq \beta \text { in } A \text { implies } \mathcal{B}_{\alpha} \subset \mathcal{B}_{\beta} \text {. }
$$

The following proposition shows how topological behaviors arise from controlaffine input-output systems.

Proposition 3.3. Consider system (2) and assume that there is a real function $\gamma(t) \geq 1, t \in \mathbb{R}$, which is locally Lebesgue integrable with the following property: For every compact set $U \times K \subset \mathbb{R}^{m} \times \mathbb{R}^{n}$ there is $\alpha_{U, K}>0$ such that for all $u \in \mathcal{U}$ and $x \in K$

$$
|h(\varphi(t, x, u), u(t))| \leq \gamma(t) \alpha_{U, K} \text { for almost all } t \in \mathbb{R} .
$$

Define a measure $\mu$ by $\mu=\gamma(\cdot)^{-1} \lambda$, where $\lambda$ denotes the Lebesgue measure. Then

$$
\mathcal{B}=\left\{(u, y) \in L_{\infty}\left(\mathbb{R}, \mathbb{R}^{m} \times \mathbb{R}^{k}, \mu\right), \begin{array}{c}
u \in L_{\infty}\left(\mathbb{R}, \mathbb{R}^{m}\right) \text { and there is } x \in \mathbb{R}^{n} \\
\text { with } y(t)=h(\varphi(t, x, u), u(t)) \text { for } t \in \mathbb{R}
\end{array}\right\}
$$

is a topological behavior with filtration given by the sets $\mathcal{B}_{U \times K}$ defined in Proposition 2.4 and $A=\left\{U \times K, U \in \mathrm{Co}_{0}\left(\mathbb{R}^{m}\right)\right.$ and $\left.K \in \operatorname{co}\left(\mathbb{R}^{n}\right)\right\}$ quasi-ordered by inclusion.

Proof. Observe that the assumptions on $\gamma$ guarantee that $0<\gamma(t)^{-1} \leq 1$ and hence $L_{p}\left(\mathbb{R}, \mathbb{R}^{m}\right) \subset L_{p}\left(\mathbb{R}, \mathbb{R}^{m}, \gamma(\cdot)^{-1} \lambda\right), p=1, \infty$. Furthermore note that for every $x \in \mathbb{R}^{n}$ and $u \in L_{\infty}\left(\mathbb{R}, \mathbb{R}^{m}\right)$ the output satisfies $y \in L_{\infty}\left(\mathbb{R}, \mathbb{R}^{k}, \mu\right)$, since

$$
|y(t)| \gamma(t)^{-1}=|h(\varphi(t, x, u), u(t))| \gamma(t)^{-1} \leq \alpha_{U, K},
$$

where $U$ is taken as the ball around the origin with radius $\|u\|_{\infty}$. Invariance of $\mathcal{B}$ is obvious by definition. For a compact and convex subset $U$ the set $\mathcal{U}$ is a weak* compact subset of $L_{\infty}\left(\mathbb{R}, \mathbb{R}^{m}, \mu\right)$. Then the other assertions follow from Proposition 2.4.

The following example shows that observed linear control systems define a topological behavior in the sense above.

Example 3.4. Consider

$$
\dot{x}(t)=A x(t)+B u(t), y(t)=C x(t)+D u(t)
$$

with matrices $A, B, C, D$ of appropriate dimensions. Let $\lambda_{\max }:=\max \{|\operatorname{Re} \nu|\}$, where the maximum is taken over the eigenvalues $\nu$ of $A$. There is a constant $c_{0}>0$ such that, by the variations-of-constants formula,

$$
\left\|\varphi\left(t, x_{0}, u\right)\right\| \leq c_{0} e^{\lambda_{\max }|t|}\left[\left\|x_{0}\right\|+\|B\|\|u\|_{\infty}\right]+\|B\|\|u\|_{\infty}, t \in \mathbb{R} .
$$


Since

$$
|h(\varphi(t, x, u), u(t))| \leq\|C\|\left\|\varphi\left(t, x_{0}, u\right)\right\|+\|D\|\|u(t)\|,
$$

this furnishes the desired estimate (13) with $\gamma(t)=\max \left\{1, e^{\lambda_{\max }|t|}\right\}$ and

$$
\alpha_{U, K}=\max \left\{1, c_{0}\|C\|\left[\left\|x_{0}\right\|+\|B\|\|u\|\right]+\|D\|\|u\|, x_{0} \in K \text { and } u \in U\right\} .
$$

Thus Proposition 3.3 describes the input-output behavior of the system (14).

For a topological behavior $\mathcal{B}$, one can study the controllability properties in each subset $\mathcal{B}_{\alpha}$. We denote the corresponding objects by an index $\alpha$, e.g., $\mathcal{O}^{\alpha,+}(v)$ denotes the positive orbit in $\mathcal{B}_{\alpha}$. The condition of regular growth formulated below allows us to show that, generically, control sets and chain control sets coincide.

Definition 3.5. For a topological behavior $\mathcal{B}$, let $\alpha \in A$. A function $v \in \mathcal{B}_{\alpha}$ is called inner if there is $T>0$ such that for all $\beta>\alpha$ there is $\varepsilon>0$ such that

$$
\mathrm{d}^{+}\left(w, \Theta_{T} v\right)<\varepsilon \text { in } \mathcal{B}_{\alpha} \text { implies } w \in \mathcal{O}_{\leq T}^{\beta,+}(v) .
$$

A behavior $\left\{\mathcal{B}_{\alpha}\right\}_{\alpha \in A}$ has regular growth if for all $\alpha \in A$ the elements of $\mathcal{B}_{\alpha}$ are uniformly inner, i.e., one may choose $\varepsilon=\varepsilon(\beta, T)$ independent of $v \in \mathcal{B}_{\alpha}$.

The following proposition shows that for control affine systems the regular growth condition reduces to a condition in the state space.

Proposition 3.6. Consider a topological behavior associated to a control-affine system as in Proposition 3.3 with $h(x, u)=x$ and filtration given by

$$
\{\rho \cdot U, \rho \geq 0\} \times K
$$

where $U \in \mathrm{Co}_{0}\left(\mathbb{R}^{m}\right)$ and $K \in \operatorname{co}\left(\mathbb{R}^{n}\right)$ are fixed (thus $A=\{\rho \geq 0\}$ ). Then for $(u, x) \in \rho \cdot \mathcal{U} \times K, \rho \geq 0$, with $\varphi(\cdot, x, u) \subset K$, the following conditions are equivalent:

(i) The input-state pair $v=(u, \varphi(\cdot, x, u)) \in \mathcal{B}_{\rho}$ is inner.

(ii) There is $T>0$ such that for all $\rho^{\prime}>\rho$

$$
\varphi(T, x, u) \in \operatorname{int}\left\{\varphi\left(t, x, u^{\prime}\right), 0 \leq t \leq T \text { and } u^{\prime} \in \rho^{\prime} \cdot \mathcal{U}\right\} .
$$

Proof. Suppose that (ii) holds. Let $\varepsilon_{k} \searrow 0$ and consider $w_{k}=\left(u_{k}, \varphi\left(\cdot, x_{k}, u_{k}\right)\right) \in \mathcal{B}_{\rho}$ with

$$
\mathrm{d}^{+}\left(w_{k}, \Theta_{T} v\right)<\varepsilon_{k}
$$

We claim that $x_{k} \rightarrow \varphi(T, x, u)$. In fact, for every subsequence there is $x^{*} \in K$ with $x_{k} \rightarrow x^{*}$. Now d $\mathrm{d}^{+}\left(w_{k}, \Theta_{T} v\right) \rightarrow 0$ implies weak* convergence $u_{k} \rightarrow u(T+\cdot)$ on $[0, \infty)$ and hence uniform convergence on bounded intervals of $\varphi\left(t, x_{k}, u_{k}\right)$ to $\varphi\left(t, x^{*}, u(T+\cdot)\right)$. This implies weak* convergence, and hence it follows that

$$
\mathrm{d}^{+}\left(w_{k}, v^{*}\right) \rightarrow 0 \text { with } v^{*}:=\left(x^{*}, u(T+\cdot)\right) .
$$

Since the limit is unique on $[0, \infty)$, we obtain $x^{*}=\varphi(T, x, u)$ as claimed. We conclude, using (ii), that for $\varepsilon_{k}>0$, small enough, $\mathrm{d}^{+}\left(w_{k}, \Theta_{T} v\right)<\varepsilon_{k}$ implies that $x_{k}=\varphi\left(t, x, u^{\prime}\right)$ for some $t \in[0, T]$ and some $u^{\prime} \in \rho^{\prime} \cdot \mathcal{U}$. Thus $w_{k} \in \mathcal{O}_{\leq T}^{\rho^{\prime}+}(v)$, and hence (i) follows.

Conversely, suppose that (i) holds and let $\rho^{\prime}>\rho$. Then there is $\varepsilon>0$ such that $\mathrm{d}^{+}\left(w, \Theta_{T} v\right)<\varepsilon$ in $\mathcal{B}_{\rho}$ implies $w \in \mathcal{O}_{\leq T}^{\rho^{\prime},+}(v)$. There is $\delta>0$ such that $|y-\varphi(T, x, u)|<\delta$ implies for $w:=(u(T+\cdot), \varphi(\cdot, y, u))$ that

$$
\mathrm{d}^{+}(w,(u(T+\cdot), \varphi(T+\cdot, x, u)))=\mathrm{d}^{+}\left(w, \Theta_{T} v\right)<\varepsilon .
$$

Thus $w \in \mathcal{O}_{\leq T}^{\rho^{\prime},+}(v)$ and hence $y=\varphi\left(t, x, u^{\prime}\right)$ for some $0 \leq t \leq T$ and $u^{\prime} \in \rho^{\prime} \cdot \mathcal{U}$, proving (ii). 
Remark 3.7. Condition (ii) in Proposition 3.6 has been used in order to analyze the relation between control sets and chain control sets for control-affine systems. Gayer [7] shows that it is satisfied for a large class of control systems.

We return to general topological behaviors $\mathcal{B}$. Let $\rho \mapsto \alpha(\rho):\left[\rho_{*}, \rho^{*}\right) \rightarrow A$, $\rho_{*}<\rho^{*}$, be an increasing map. Then for all $v \in \mathcal{B}_{\alpha_{0}}$ the maps $\alpha \mapsto \operatorname{clO}^{\alpha,+}(v)$ and $\alpha \mapsto \mathcal{O}_{c}^{\alpha,+}(v)$, defined for $\alpha \geq \alpha_{0}$, are increasing, where $\operatorname{cl} S$ denotes the closure of a set $S$.

Lemma 3.8. Let $\rho \mapsto \alpha(\rho):\left[\rho_{*}, \rho^{*}\right) \rightarrow A, \rho_{*}<\rho^{*}$, be an increasing map. Then for all but at most countably many $\rho$-values the maps $\rho \mapsto \operatorname{cl}^{\alpha(\rho),+}(v)$ and $\rho \mapsto$ $\mathcal{O}_{c}^{\alpha(\rho),+}(v)$ are increasing and continuous.

Proof. Monotonicity is obvious. The continuity assertion follows from Scherbina's Lemma (see Scherbina [14] or Pilyugin [9]), which states that increasing, compactvalued mappings defined on $[0, \infty)$ are continuous with respect to the Hausdorff metric for all but at most countably many $\rho$-values.

Proposition 3.9. Let $\alpha \in A$ and suppose that the elements of $\mathcal{B}_{\alpha}$ are uniformly inner. Then $\mathcal{O}_{c}^{\alpha,+}(v) \subset \mathcal{O}^{\beta,+}(v)$ for all $v \in \mathcal{B}_{\alpha}$ and for all $\beta>\alpha$.

Proof. Let $\beta>\alpha$. Then there is $\varepsilon>0$ such that for $v \in \mathcal{B}_{\alpha}$

$$
\mathrm{d}^{+}\left(w, \Theta_{T} v\right)<\varepsilon \text { in } \mathcal{B}_{\alpha} \text { implies } w \in \mathcal{O}_{\leq T}^{\beta,+}(v) .
$$

Thus the assertion follows since $\varepsilon>0$ does not depend on the considered element of $\mathcal{B}_{\alpha}$.

Remark 3.10. For the state space theory, [3, Lemma 4.5.5] uses local accessibility in a crucial way in order to show that inner pairs in an invariant compact set are uniformly inner.

If $\mathcal{E}^{\alpha}$ is a (behavioral) chain control set and $\beta>\alpha$, then there exists a unique chain control set $\mathcal{E}^{\beta}$ of $\mathcal{B}_{\beta}$ containing $\mathcal{E}^{\alpha}$; similarly for control sets. With this notation, the following results hold.

Lemma 3.11. Let $\rho \mapsto \alpha(\rho):\left[\rho_{*}, \rho^{*}\right) \rightarrow A, \rho_{*}<\rho^{*}$, be a strictly increasing map. Then the corresponding control sets and chain control sets satisfy for all but at most countably many $\rho$-values

$$
\mathcal{E}^{\alpha(\rho)}=\bigcap_{\rho^{\prime}>\rho} \mathcal{E}^{\alpha\left(\rho^{\prime}\right)} \text { and } \mathrm{cl} \mathcal{D}^{\alpha(\rho)}=\bigcap_{\rho^{\prime}>\rho} \operatorname{cl} \mathcal{D}^{\alpha\left(\rho^{\prime}\right)} .
$$

Proof. This again is Scherbina's Lemma.

The next theorem shows that under the assumption of regular growth, generically, the chain orbits are the closures of orbits, and, similarly, the chain control sets are the closures of control sets.

Theorem 3.12. Suppose that $\mathcal{B}$ is a topological behavior with regular growth and let $\rho \mapsto \alpha(\rho):\left[\rho_{*}, \rho^{*}\right) \rightarrow A, \rho_{*}<\rho^{*}$, be a strictly increasing map. Then, abbreviating for the chain control sets and the control sets $\mathcal{E}^{\rho}:=\mathcal{E}^{\alpha(\rho)}$ and $\mathcal{D}^{\rho}:=\mathcal{D}^{\alpha(\rho)}$, respectively, one has

(i) for all chain control sets $\mathcal{E}^{\rho_{*}}$ there are chain control sets $\mathcal{E}^{\rho}$ with $\mathcal{E}^{\rho^{*}} \subset \mathcal{E}^{\rho}$ for all $\rho>\rho_{*}$;

(ii) for all but at most countably many $\rho$-values the chain orbits are the closures of orbits and the chain control sets $\mathcal{E}^{\rho}$ are the closures of control sets $\mathcal{D}^{\rho}$. 
Proof. Assertion (i) is obvious. For assertion (ii) observe that $\rho<\rho^{\prime}$ implies $\mathcal{E}^{\rho} \subset$ $\mathcal{D}^{\rho^{\prime}}$, since $\mathcal{E}^{\rho} \subset \mathcal{O}_{c}^{\rho,+}(v) \subset \mathcal{O}^{\rho^{\prime},+}(v)$ for all $v \in \mathcal{E}^{\rho}$. Furthermore for all but at most countably many $\rho$-values

$$
\operatorname{cl}^{\rho} \subset \mathcal{E}^{\rho} \subset \bigcap_{\rho^{\prime}>\rho} \mathcal{D}^{\rho^{\prime}} \subset \bigcap_{\rho^{\prime}>\rho} \mathcal{E}^{\rho^{\prime}} \subset \bigcap_{\rho^{\prime}>\rho} \mathcal{D}^{\rho^{\prime}}=\mathrm{cl}^{\rho}
$$

here the fourth inclusion follows since one finds for $\rho^{\prime}>\rho$ a $\rho^{\prime \prime}$ with $\rho^{\prime}>\rho^{\prime \prime}>\rho$ and hence $\alpha\left(\rho^{\prime}\right)>\alpha\left(\rho^{\prime \prime}\right)>\alpha(\rho)$.

Remark 3.13. A linear behavior is given by a topological behavior on a vector bundle $\mathcal{V}$ for which the time shifts are linear flows (for each $\alpha \in A$ ). An example are bilinear control systems (without output): As a special case of Proposition 3.3. consider

$$
\dot{x}(t)=\left[A_{0}+\sum_{i=1}^{m} u_{i}(t) A_{i}\right] x(t), y(t)=x(t),
$$

with matrices $A_{i} \in \mathbb{R}^{n \times n}$. Then the input-state pairs

$$
\left\{(u, x) \in L_{\infty}\left(\mathbb{R}, \mathbb{R}^{m} \times \mathbb{R}^{n}, \mu\right), \text { there is } x_{0} \in \mathbb{R}^{n} \text { with } x(t)=\varphi\left(t, x_{0}, u\right) \text { for } t \in \mathbb{R}\right\}
$$

may be considered as a vector bundle over the chain transitive base space $\mathcal{U}$ (here the measure $\mu$ has a density with respect to Lebesgue measure determined by the maximal and and minimal Lyapunov exponents). Hence Selgrade's Theorem (see Salamon and Zehnder for a proof [13]) implies that there are at most $n$ chain control sets in the projective bundle; they form the finest Morse decomposition.

Next we show that a behavioral control set of an input-output system (2) uniquely determines a control set in the state space, if the input-output pair determines the state trajectory.

Proposition 3.14. Consider a set $\mathcal{B}_{U \times K} \subset L_{\infty}\left(\mathbb{R}, \mathbb{R}^{m} \times \mathbb{R}^{k}, \mu\right)$ of input-output pairs as in Proposition 2.4 with $U \in \mathrm{Co}_{0}\left(\mathbb{R}^{m}\right)$ and $K \in \mathrm{co}\left(\mathbb{R}^{n}\right)$, and assume that for all $(u, y) \in \mathcal{B}_{U \times K}$ and all $T>0$ there is a unique point $x \in \mathbb{R}^{n}$ such that

$$
y(t)=h(\varphi(t, x, u), u(t)), t \in[0, T] .
$$

Let $\mathcal{D} \subset \mathcal{B}_{U \times K}$ be a (behavioral) control set. Then there is a unique control set $D \subset$ $\mathbb{R}^{n}$ such that $(u, y) \in \mathcal{D}$ implies that there is $x \in D$ with $y(t)=h(\varphi(t, x, u), u(t))$ for all $t \in \mathbb{R}$.

Proof. For $i=0,2$ let $\left(u^{i}, y^{i}\right) \in \mathcal{D}$. Then there are unique $x^{i} \in \mathbb{R}^{n}$ such that for all $t$

$$
y^{i}(t)=h\left(\varphi\left(t, x^{i}, u^{i}\right), u^{i}(t)\right)
$$

One can control from $\left(u^{0}, y^{0}\right)$ to $\left(u^{2}, y^{2}\right)$, i.e., one finds $\left(u^{1}, y^{1}\right) \in \mathcal{D}$ and $T>0$ with

$$
\left(u^{1}(t), y^{1}(t)\right)= \begin{cases}\left(u^{0}(t), y^{0}(t)\right) & \text { for } t \leq 0 \\ \left(u^{2}(t-T), y^{2}(t-T)\right) & \text { for } t \geq T .\end{cases}
$$

There is a unique $x^{1} \in \mathbb{R}^{n}$ with

$$
y^{1}(t)=h\left(\varphi\left(t, x^{1}, u^{1}\right), u^{1}(t)\right) \text { for all } t \in \mathbb{R} .
$$


Since, by our assumption, the corresponding point in $\mathbb{R}^{n}$ is already determined by any time interval, this implies that

$$
x^{1}=x^{0} \text { and } \varphi\left(T, x^{1}, u^{1}\right)=x^{2} .
$$

Reversing the roles of $\left(u^{0}, y^{0}\right)$ and $\left(u^{2}, y^{2}\right)$, one sees that $x^{0}$ and $x^{1}$ lie in a control set $D \subset \mathbb{R}^{n}$. Using shift invariance of $\mathcal{D}$, one also sees that $\varphi\left(t, x^{0}, u^{0}\right) \in D$ for all $t \in \mathbb{R}$. Hence the inclusion follows. Uniqueness is clear.

Remark 3.15. If local accessibility holds for the system (2) in the state space $\mathbb{R}^{n}$, then one has exact controllability in the interior of a control set in the state space. Thus for all $x$ in the interior of a control set $D$ in $\mathbb{R}^{n}$ there is a control set $\mathcal{D}$ with

$$
\varphi(t, x, u) \in \operatorname{int} D \text { and } y(t)=h(\varphi(t, x, u), u(t)) \text { for all } t \in \mathbb{R} \text { implies }(u, y) \in \mathcal{D} \text {. }
$$

Remark 3.16. The assumption of Proposition 3.14 holds, e.g., for systems of the type

$$
\ddot{x}(t)+g_{0}(x(t)) \dot{x}(t)+g_{1}(x(t))=u(t), y(t)=x(t)
$$

with smooth real functions $g_{0}, g_{1}$. Here on any nontrivial interval the function $y(t)=x(t)$ determines the state $(x(t), \dot{x}(t))$ and hence the initial state $x \in \mathbb{R}^{2}$.

We add a simple example, where the behavioral control set is not just the projected lift of a control set in the state space.

Example 3.17. Consider an observed affine control system

$$
\dot{x}(t)=A x(t)+B u(t)+d, u(t) \in U, y(t)=C x(t) .
$$

Suppose that

$$
A=\left(\begin{array}{ll}
A_{1} & 0 \\
0 & I_{n-k}
\end{array}\right), B=\left(\begin{array}{l}
B_{1} \\
0
\end{array}\right), C=\left(\begin{array}{ll}
I_{k} & 0
\end{array}\right), d=\left(\begin{array}{l}
0 \\
d_{2}
\end{array}\right) .
$$

Assume that $\left(A_{1}, B_{1}\right) \in \mathbb{R}^{k \times k} \times \mathbb{R}^{k \times m}$ is controllable and $0 \neq d_{2} \in \mathbb{R}^{n-k}$, and $U$ is compact and convex with $0 \in \operatorname{int} U$. The trajectories of (15) with initial condition $x(0)=\left(x_{1}^{0}, x_{2}^{0}\right)^{T}$ are given by

$$
\begin{aligned}
\varphi(t, x, u) & =\left(\begin{array}{l}
e^{A_{1} t} x_{1}^{0} \\
e^{t} x_{2}^{0}
\end{array}\right)+\int_{0}^{t}\left(\begin{array}{l}
e^{A_{1}(t-s)} B_{1} u(s) \\
e^{(t-s)} d_{2}
\end{array}\right) d s \\
& =\left(\begin{array}{l}
e^{A_{1} t} x_{1}^{0}+\int_{0}^{t} e^{A_{1}(t-s)} B_{1} u(s) d s \\
e^{t} x_{2}^{0}+\left(e^{t}-1\right) d_{2}
\end{array}\right) .
\end{aligned}
$$

This system has no control set in the state space, since at least one of the last $n-k$ components is strictly monotone. On the other hand, the linear system

$$
\dot{x}_{1}(t)=A_{1} x_{1}(t)+B_{1} u(t), u(t) \in U,
$$

has a unique control set $D_{1} \subset \mathbb{R}^{k}$. Denoting the trajectories of (16) by $\varphi_{1}\left(t, x_{1}, u\right)$ one obtains for all $\left(x_{1}, x_{2}\right)$

$$
\varphi_{1}\left(t, x_{1}, u\right)=e^{A_{1} t} x_{1} \int_{0}^{t} e^{A_{1}(t-s)} B_{1} u(s) d s=C \varphi\left(t,\left(x_{1}, x_{2}\right), u\right) .
$$


Thus it easily follows that the subset $\mathcal{D} \subset L_{\infty}\left(\mathbb{R}, \mathbb{R}^{m+k}, \mu\right)$ (with $\mu$ as in Proposition 3.3 and Example 3.4), defined by

$\mathcal{D}:=\left\{(u, y)\right.$, there is $\left(x_{1}, x_{2}\right) \in D_{1} \times \mathbb{R}^{n-k}$ with $y(t)=C \varphi\left(t,\left(x_{1}, x_{2}\right), u\right)$ for $\left.t \in \mathbb{R}\right\}$,

is a behavioral control set for system (15).

System (15) is not locally accessible. However, it can be easily modified in the following way: Replace $B$ and the control range by

$$
B=\left(\begin{array}{ll}
B_{1} & 0 \\
0 & I_{k}
\end{array}\right) \text { and } U \times[0,1]^{k},
$$

respectively. Then the resulting system is locally accessible, but has no control set in the state space. The other arguments remain valid.

Remark 3.18. Using the positive orbits as in Definition 2.2, one might introduce a notion of accessibility of behaviors, as the property that the interior of $\mathcal{O}^{+}(v)$ is nonvoid. The consequences of such a property remain to be explored.

\section{Conclusions}

The present paper introduces a topological notion of behaviors by considering the time shift on $L_{\infty}$-spaces. Subsets of complete controllability and chain controllability are studied. While controllability is not, in general, a notion of the corresponding topological dynamical system, chain controllability turns out to be equivalent to chain transitivity. This is obtained by an appropriate generalization of results from the theory of continuous-time control-affine state space systems.

\section{REFERENCES}

[1] M. V. Bebutov, Dynamical systems in the space of continuous functions, Dokl. Akad. Nauk SSSR, 27 (1940), pp. 904-906. In Russian.

[2] F. Colonius and W. Kliemann, Some aspects of control systems as dynamical systems, J. Dyn. Diff. Equations, 5 (1993), pp. 469-494. MR/1235039 (94g:93063)

[3] — The Dynamics of Control, Birkhäuser, Boston, 2000.

[4] Behaviors and controllability, in Electronic Proceedings of the 16th Conference on Mathematical Theory of Networks and Systems (MTNS), July 5-9, 2004, Leuven (Belgium), 2004.

[5] F. Colonius and M. Spadini, Uniqueness of local control sets, J. Dynamical and Control Systems, 9 (2003), pp. 513-530. MR2001957 (2004f:93010)

[6] R. Easton, Geometric Methods for Discrete Dynamical Systems, Oxford University Press, 1998. MR1616726 (2000e:37002)

[7] T. Gayer, Control sets and their boundaries under parameter variation, J. Diff. Equations, 201 (2004), pp. 177-200. MR2057543 (2005g:93063)

[8] M. Golubitsky AND D. Schaeffer, Singularities and Groups in Bifurcation Theory, Springer-Verlag, 1985. MR771477 (86e:58014)

[9] S. Pilyugin, The Space of Dynamical Systems with the $C^{0}$-Topology, Springer-Verlag, 1994. MR.1329732 (96g:58049)

[10] J. W. Polderman And J. C. Willems, Introduction to Mathematical Systems Theory. A Behavioral Approach, Springer-Verlag, 1997. MR1480665 (99f:93001)

[11] A. B. Poore, A model equation arising from chemical reactor theory, Arch. Rational Mech. Anal., 52 (1974), pp. 358-388. MR0338508 (49:3272)

[12] C. Robinson, Dynamical Systems. Stability, Symbolic Dynamics, and Chaos, CRC Press Inc., 1995. MR1396532(97e:58064)

[13] D. Salamon AND E. Zehnder, Flows on vector bundles and hyperbolic sets, Trans. Amer. Math. Soc., 306 (1988), pp. 623-649. MR933310 (89f:58112)

[14] N. Scherbina, Continuity of one-parameter families of sets, Dokl. Ak. Nauk SSSR, 234 (1977), pp. 327-329. In Russian. MR0440500 (55:13375) 
[15] G. R. SELL, Topological Dynamics and Ordinary Differential Equations, Van Nostrand Reinhold, London, 1971. MR0442908 (56:1283)

[16] D. Szolnoki, Set oriented methods for computing reachable sets and control sets, Discrete and Continuous Dynamical Systems-Series B, 3 (2003), pp. 361-382. MR.1974152 (2004a:93007)

[17] J. C. Willems, Models for dynamics, in Dynamics Reported, Vol. 2, U. Kirchgraber and H. Walther, eds., Teubner, 1989, pp. 171-269. MR1000978 (90g:58121)

Institut für Mathematik, Universität Augsburg, 86135 Augsburg, Germany

E-mail address: fritz.colonius@math.uni-augsburg.de

Department of Mathematics, Iowa State University, Ames, Iowa 50011

E-mail address: kliemann@iastate.edu 